\title{
Spectroscopy on the proton drip-line: Probing the structure dependence of isospin nonconserving interactions
}

\author{
J. Henderson, ${ }^{1}$ D. G. Jenkins, ${ }^{1}$ K. Kaneko, ${ }^{2}$ P. Ruotsalainen,${ }^{3}$ P. Sarriguren,${ }^{4}$ K. Auranen, ${ }^{3}$ M. A. Bentley, ${ }^{1}$ \\ P. J. Davies, ${ }^{1}$ A. Görgen, ${ }^{5}$ T. Grahn, ${ }^{3}$ P. T. Greenlees, ${ }^{3}$ A. Hay, ${ }^{1}$ T. W. Henry, ${ }^{1}$ A. Herzáň, ${ }^{3}$ U. Jakobsson, ${ }^{3}$ R. Julin, ${ }^{3}$ \\ S. Juutinen, ${ }^{3}$ J. Konki, ${ }^{3}$ M. Leino, ${ }^{3}$ C. McPeake, ${ }^{6}$ S. Milne, ${ }^{1}$ A. J. Nichols, ${ }^{1}$ J. Pakarinen, ${ }^{3}$ P. Papadakis, ${ }^{3}$ J. Partanen, ${ }^{3}$ \\ P. Peura, ${ }^{3}$ P. Rahkila, ${ }^{3}$ E. Sahin, ${ }^{5}$ M. Sandzelius, ${ }^{3}$ J. Sarén, ${ }^{3}$ C. Scholey, ${ }^{3}$ M. Siciliano, ${ }^{3,7}$ L. Sinclair, ${ }^{1}$ J. Sorri, ${ }^{3}$ S. Stolze, ${ }^{3}$ \\ J. Uusitalo, ${ }^{3}$ R. Wadsworth, ${ }^{1}$ and M. Zielińska ${ }^{8}$ \\ ${ }^{1}$ Department of Physics, University of York, Heslington, York YO10 5DD, United Kingdom \\ ${ }^{2}$ Department of Physics, Kyushu Sangyo University, Fukuoka 813-8503, Japan \\ ${ }^{3}$ Department of Physics, University of Jyväskylä, FIN-40014 Finland \\ ${ }^{4}$ Instituto de Estructura de la Materia, CSIC, Serrano 123, E-28006 Madrid, Spain \\ ${ }^{5}$ Department of Physics, University of Oslo, N-0316 Oslo, Norway \\ ${ }^{6}$ Oliver Lodge Laboratory, University of Liverpool, Liverpool L69 7ZE, United Kingdom \\ ${ }^{7}$ Dipartimento di Fisica e Astronomia, Universitá di Padova, I-35131 Padova, Italy \\ ${ }^{8}$ CEA Saclay, IRFU/Service de Physique Nucléaire, F-91191 Gif-sur-Yvette, France
}

(Received 26 May 2014; revised manuscript received 10 October 2014; published 18 November 2014)

\begin{abstract}
The recoil- $\beta$ tagging technique was used to identify transitions associated with the decay of the $2^{+}$and, tentatively, the $4^{+}$excited states in ${ }^{74} \mathrm{Sr}$. Combining these results with published data for the $A=74$ isobars, triplet energy differences (TEDs) have been extracted, the heaviest case for which these values have been evaluated. State-of-the-art shell-model calculations using the JUN45 interaction and incorporating a $J=0$ isospin nonconserving (INC) interaction with an isotensor strength of $100 \mathrm{keV}$ can reproduce the trend in the TED data, with particularly good agreement for the $2^{+}$state. This agreement for the TED data taken together with the fact that agreement has also been shown between shell-model calculations with the same strength of INC interaction in the $f_{7 / 2}$ shell and recently for $A=66$ strongly suggests that such an interaction exists throughout the nuclear chart and cannot have a strong dependence on details of nuclear structure such as which nuclear orbitals are occupied. It also supports the hypothesis that only a $J=0$ component of the INC interaction need be included to explain the observed TEDs.
\end{abstract}

DOI: 10.1103/PhysRevC.90.051303

PACS number(s): 21.10.Re, 21.60.Cs, 23.20.Lv, 27.50.+e

Isospin symmetry is a central concept in nuclear physics, based on the idea that the proton and neutron are two isospin states of a single object, the nucleon. This would imply an identical pattern of excited states across a $T=1$ isospin triplet. These symmetries are broken due to a number of nuclear structure effects, most importantly the fact that the proton carries an electric charge while the neutron does not. When exploring such breakdown of symmetries, it is instructive for the study of isospin triplets, to evaluate triplet energy differences (TEDs) as a function of spin $J$ defined by

$$
\mathrm{TED}_{J}=E_{J, T, T_{2}=-1}^{*}+E_{J, T, T_{2}=+1}^{*}-2 E_{J, T, T_{z}=0}^{*} .
$$

Such isotensor energy differences are essentially independent of single-particle effects, which makes them particularly simple. Since contributions involving Coulomb effects are readily calculable, TEDs are particularly sensitive to additional terms such as isospin nonconserving (INC) components. It has been shown that such a component is necessary to reproduce experimental mirror-energy differences (MEDs) in the $f_{7 / 2}$ shell [1]. Indeed, recent work identifying the $\left(4_{1}^{+}\right)$and $\left(6_{1}^{+}\right)$ excited states in the $N=Z-2$ nucleus ${ }^{66} \mathrm{Se}$ [2] has allowed the evaluation of the TEDs for $A=66$, the first case above the ${ }^{56} \mathrm{Ni}$ closed shell; and it appears that an INC term is also mandatory in reproducing the TED. Nucleon-nucleon scattering data have previously been used to infer the INC component to the nuclear interaction, the isotensor component of which is consistent with that previously observed in the $f_{7 / 2}$ orbital [3]. The measurement of such a component in-medium is of considerable interest, and it is not yet clear whether the INC component arising from energy-difference measurements is indeed the same as that determined from scattering data. It is of high interest to extend such studies to higher masses, where the structure of the low-spin states involved is very different, evolving from dominance by $f p$ orbitals to $g_{9 / 2}$ orbitals by $A=74$ [4]. Moreover, predictions for the TEDs already exist with and without the INC component [5]. Irrespective of the techniques employed, however, the study of exotic proton-rich nuclei is challenging due to their low production cross sections. Allied to this is the fact they are generally produced as a small fraction of a large sample of less exotic nuclei. Sensitive techniques are needed to discriminate the exotic nuclei of interest. In the present work, we report data on excited states in ${ }^{74} \mathrm{Sr}$ for the first time.

To probe excited states in ${ }^{74} \mathrm{Sr}$, the recoil- $\beta$ tagging (RBT) technique [6,7] was employed. Instead of using a discrete decay energy such as a characteristic $\alpha$ or proton decay as a tag, RBT exploits the special characteristics of some $\beta$ decays at and beyond the line of $N=Z$, namely, their short half-life and high endpoint energy, stemming from their Fermi superallowed character. The RBT technique was initially demonstrated for the case of ${ }^{74} \mathrm{Rb}$ where excited states were well known [6]. It was later used to identify excited states in 
the $N=Z$ nucleus ${ }^{78} \mathrm{Y}$ for the first time [8]. RBT has recently been used on the $A=66$ isobar to provide in-depth $\gamma$-ray spectroscopy of the $T_{z}=0$ nucleus ${ }^{66} \mathrm{As}$ [9] and in the first observation of transitions from the $4_{1}^{+}$and $6_{1}^{+}$excited states in the $T_{z}=-1$ nucleus ${ }^{66} \mathrm{Se}$ [2]. It has also been used to extend the knowledge of excited states in ${ }^{62} \mathrm{Ga}[10]$.

A $105-\mathrm{MeV}{ }^{36} \mathrm{Ar}$ beam from the K130 cyclotron at the University of Jyväskylä was impinged onto a $0.66-\mathrm{mg} / \mathrm{cm}^{2}$ ${ }^{40} \mathrm{Ca}$ target, backed by a $42-\mu \mathrm{g} / \mathrm{cm}^{2}$ nat $\mathrm{C}$ reset foil to produce the nuclei of interest through the ${ }^{40} \mathrm{Ca}\left({ }^{36} \mathrm{Ar}, 2 n\right){ }^{74} \mathrm{Sr}$ reaction. Recoils were separated from the scattered beam using the recoil ion transport unit (RITU) gas-filled separator [11] and implanted into a 500- $\mu$ m-thick double-sided silicon strip detector (DSSD) [7]. Recoil energy was extracted from the DSSD, $\Delta E$ from the GREAT multiwire proportional counter [12] (MWPC), and time of flight (ToF) information was taken between the MWPC and DSSD. A phosphor-sandwich (phoswich) detector [7] abutted the DSSD to provide $\beta$ particle selection. The phoswich consists of a $10-\mathrm{mm}$-thick fast-plastic scintillator optically coupled to a 31.5-mm-thick slow-plastic scintillator. DSSD amplification was set such that the horizontal strips detected events with a maximum energy of $1 \mathrm{MeV}$ and the vertical strips recorded a maximum energy of approximately $10 \mathrm{MeV}$. Thresholds were set to less than $100 \mathrm{keV}$ for both the horizontal and vertical DSSD strips in order to provide pixel information for low-energy events. The University of York tube (UoYtube) - a targetposition charged-particle veto box [7]—-was used to suppress unwanted fusion evaporation channels. For the present study, the UoYtube single-proton veto efficiency was found to be $66 \%$. JUROGAM II, comprising 24 Eurogam II type composite clovers [13] and 15 Eurogam phase I [14] and GASP [15] type detectors, was used to detect emitted $\gamma$ rays at the target position. Data were recorded for $135 \mathrm{~h}$ with beam intensities varying between 3 and 5 pnA. Data were acquired by the JYFL total data readout (TDR) system [16], and analyzed using the GRAIN package [17].

In the analysis, recoils were selected using appropriate conditions on $\Delta E, E$, and ToF. Prompt $\gamma$ rays were selected using recoil-ToF and the $\gamma$-ray detection time with respect to implantation. DSSD events which did not pass the recoil gates but which occurred within $100 \mathrm{~ms}$ of a valid recoil in the same pixel were considered as possible $\beta$-decay events. Fast and slow elements of the phoswich signal were deconvoluted as described in Ref. [7]. High-energy positrons were identified by gates on the energy recorded in the fast and slow parts of the phoswich. The analysis of the data was assisted by a recently obtained half-life for ${ }^{74} \mathrm{Sr}$ stemming from an experiment carried out at the RIKEN radioactive-isotope beam factory (RIBF) [18]. To search for candidate ${ }^{74} \mathrm{Sr}$ transitions, events were selected where a high-energy (>5 MeV) positron detected in the DSSD-phoswich telescope was correlated with implantation events in the same DSSD pixel within $100 \mathrm{~ms}$ (approximately three half-lives for ${ }^{74} \mathrm{Sr}$ ), and to reject all events with a coincident charged particle detected in UoYtube [see Fig. 1(a)] in order to select events corresponding to $2 n$ evaporation, i.e., ${ }^{74} \mathrm{Sr}$. This spectrum was still, however, dominated by ${ }^{74} \mathrm{Rb}$ due to false correlations related to the high

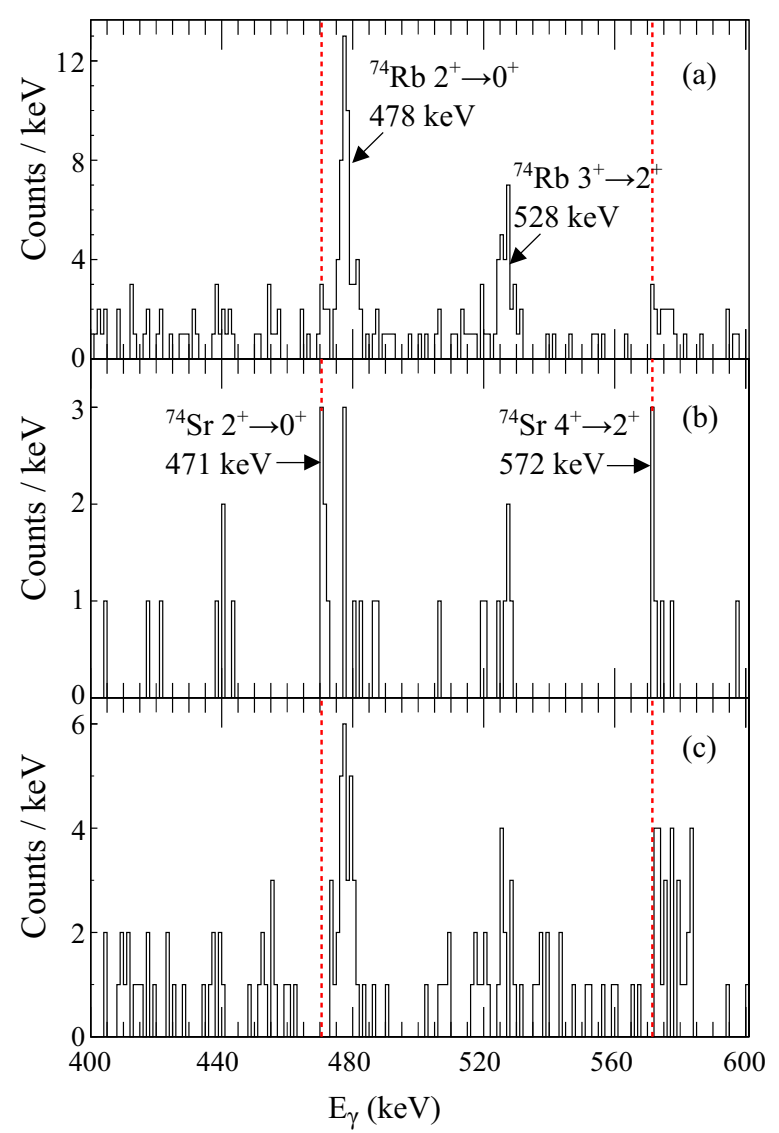

FIG. 1. (Color online) $\gamma$-ray spectrum obtained with JUROGAM II for a range of tagging conditions: (a) requiring that a decay event occurs within $100 \mathrm{~ms}$ of the correlated implantation, has a high-energy $(>5 \mathrm{MeV}$ ) positron recorded in the phoswich detector, and that no coincident charged particles were recorded in UoYtube; (b) with the same gating conditions but excluding the sections of the DSSD with high implantation rate; and (c) same conditions as (b) but for events with one or more charged particles recorded in UoYtube. The dashed lines show the location of candidate ${ }^{74} \mathrm{Sr}$ transitions.

recoil rate per pixel. Selecting the portion of the DSSD with the lowest implantation rate [see Fig. 1(b)] effectively removed the majority of the events associated with ${ }^{74} \mathrm{Rb}$, and identified two new transitions of $471(1) \mathrm{keV}$ and, tentatively, 572(1) $\mathrm{keV}$. The latter transition, in a region of near-zero background, corresponds to a statistically significant result in the $2 \sigma$ limit [19]. Under similar conditions but instead demanding that exactly one charged particle be detected in the UoYtube, these transitions are not seen [see Fig. 1(c)]. It is also possible to extract a corresponding half-life for zero charged-particle events where the 471-keV $\gamma$ ray is detected. Low statistics mean that standard fitting to a decay curve is impractical. Instead, the method of Schmidt et al. [20] was used, using the whole DSSD and a lower $\beta$-energy threshold $(3 \mathrm{MeV})$ in order to maximize statistics. A $t_{1 / 2}$ of $27(8) \mathrm{ms}$ is extracted in this manner, in good correspondence with the value measured in the RIKEN experiment [18]. As a cross-check, a half-life of 73(9) ms was extracted for events containing the 478-keV 


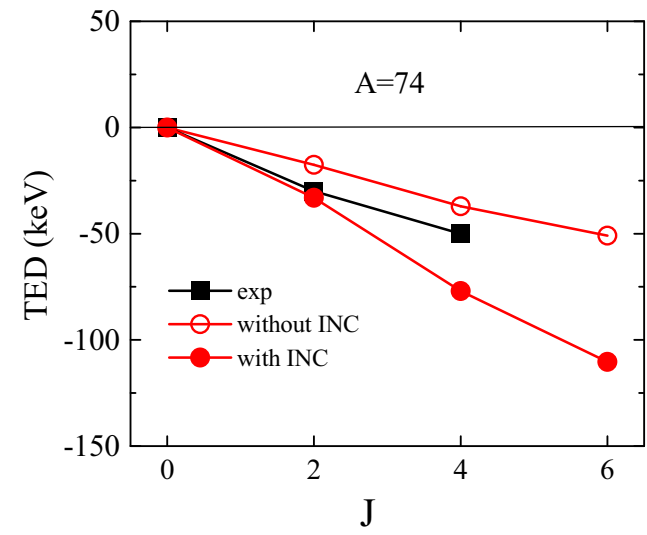

FIG. 2. (Color online) Comparison of TED for $A=74$ with the results of shell-model calculations using the JUN45 interaction (see text).

transition corresponding to the decay of the first $2^{+}$state in ${ }^{74} \mathrm{Rb}$, in conformity with the accepted value for the half-life for ${ }^{74} \mathrm{Rb}$ of $64.761(31) \mathrm{ms}$ [21]. On the basis that the transitions identified reside in the zero charged-particle emission channel and have an associated half-life consistent with that expected for ${ }^{74} \mathrm{Sr}$, it is possible to assign them to ${ }^{74} \mathrm{Sr}$. It is clear that they are also not dissimilar in energy to the analogous transitions in the isobars ${ }^{74} \mathrm{Rb}$ and ${ }^{74} \mathrm{Kr}$.

The newly obtained data on excited states in ${ }^{74} \mathrm{Sr}$ may be combined with existing data on ${ }^{74} \mathrm{Kr}$ and ${ }^{74} \mathrm{Rb}$ to evaluate TEDs for the first time for $A=74$ (see Fig. 2). As with all previously obtained data for different isobaric triplets, the TED follows a downward slope as a function of spin. To understand the implications of the new TED data for $A=74$, large-scale shell-model calculations were performed in the $p f_{5 / 2} g_{9 / 2}$ valence space. The recently proposed Sakurai and Sugiura (SS) method [22,23] was employed, using the modern JUN45 interaction [24]. Coulomb multipole, monopole, and spin-orbit interactions with the same parameters as in earlier work $[25,26]$ were included; further details can be found in Ref. [26]. It has been shown [5] that the multipole, monopole, and spin-orbit forces are not sufficient to explain the experimental TED for $A=66$ and that the INC force is important for reproducing the observed experimental data [2]. The INC force used in the present calculation comprises an isotensor strength of $100 \mathrm{keV}$ for $J=0$ couplings for all the orbitals included in the model space, which is consistent with that required to reproduce the empirical TED of the $A=42$ triplet in the $f_{7 / 2}$ shell [27]. As one can see from Fig. 2, the calculated TEDs reproduce remarkably well the present experimental data for $J=2$. However, the calculation fails to reproduce the TEDs for $J=4$. The plot for $J=4$ is rather close to that without the INC; possible reasons for a weaker agreement in this case will be considered below. Nevertheless, the key feature of the present calculations is that they account very well for the observed TEDs at $J=2$, in common with all other studied cases such as $A=66$ and the $f_{7 / 2}$ shell. This strongly suggests that the inclusion of the INC force is mandated in all cases, and appears to have a magnitude irrespective of the details of nuclear structure, such as which orbitals are active. This is an

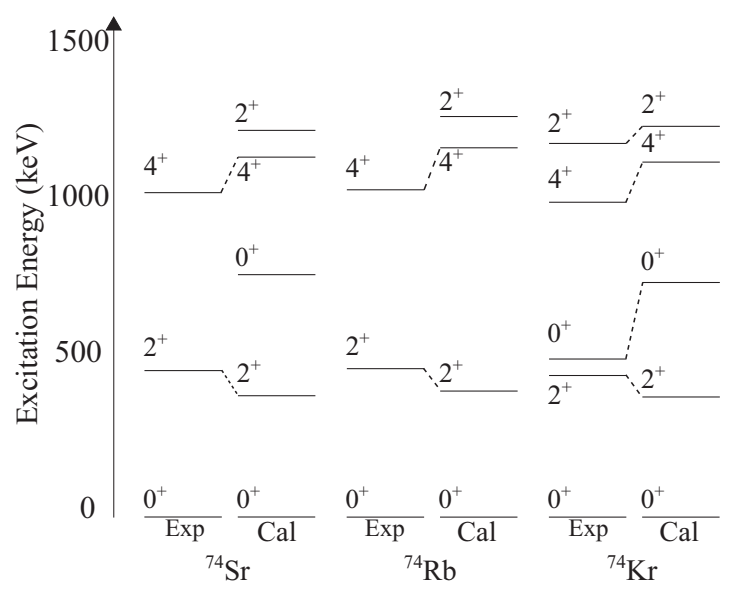

FIG. 3. Experimental and calculated level scheme for low-lying $T=1$ states in the $A=74$ isobaric triplet. It can be seen that the presented shell-model calculations reproduce the low-lying orbitals well, particularly in the yrast states, as well as reproducing the lowlying excited $0^{+}$state observed in ${ }^{74} \mathrm{Kr}$. Note that no excited $0^{+}$state could be calculated for ${ }^{74} \mathrm{Rb}$ due to $T=0$ states being present at similar excitation energy.

important insight into the nature of the INC force. In addition, the state of the art shell-model calculations correctly predict the existence of a low-lying $0^{+}$state in the $T_{z}=+1$ nuclide ${ }^{74} \mathrm{Kr}$, as shown in Fig. 3. It should be noted, however, that the observed first excited $0^{+}$energy of about $500 \mathrm{keV}$ may be due to configuration mixing between the $0_{1}^{+}$and $0_{2}^{+}$states [28].

A relevant question is whether the above conclusions are affected by proximity to the proton drip-line through, e.g., the Thomas-Ehrman effect [29,30] or for weakly bound states, coupling to the continuum which produces asymmetry in the wave functions in, e.g., the proton-rich member of an isobaric triplet [31]. The systematics suggest that all the excited states observed in this work are one- and two-proton bound; the latest mass compilations [32] provide $S_{2 p}=1.47(10) \mathrm{MeV}$ where the error is dominated by the theoretical uncertainty in the ${ }^{74} \mathrm{Sr}$ mass. However, recent theoretical calculations performed using state of the art shell-model interactions and including isospin nonconserving components [33] suggest that the $T_{z}=-1$ nuclide ${ }^{74} \mathrm{Sr}$ may lie on the verge of being two-proton unbound, predicting $S_{2 p}=1.26 \mathrm{MeV}$ using the GXPF1A interaction and $S_{2 p}=0.41 \mathrm{MeV}$ using the JUN45 interaction. The latter value would make both excited states observed in ${ }^{74} \mathrm{Sr}$ two-proton unbound.

Published deformed Skyrme (SLy4) HartreeFock + BCS + QRPA calculations [34] using $Q_{\mathrm{EC}}=11.2 \mathrm{MeV}$ predict a half-life for ${ }^{74} \mathrm{Sr}$ of $t_{1 / 2}=54 \mathrm{~ms}$ assuming an oblate shape for the ground state of ${ }^{74} \mathrm{Sr}$. Clearly, the precise half-life for ${ }^{74} \mathrm{Sr}$ in the literature is around half this value. Accordingly, the influence of the $Q_{\mathrm{EC}}$ value on these calculations has been explored (see Fig. 4). To reproduce the $t_{1 / 2}$ obtained in the present work, again, assuming oblate shape for the ground state of ${ }^{74} \mathrm{Sr}$, a $Q$ value of $Q_{\mathrm{EC}}=12.2 \mathrm{MeV}$ is required (see Fig. 4). This change in $Q$ value would imply $S_{2 p} \approx 0.45 \mathrm{MeV}$, in good agreement with the prediction using the JUN45 


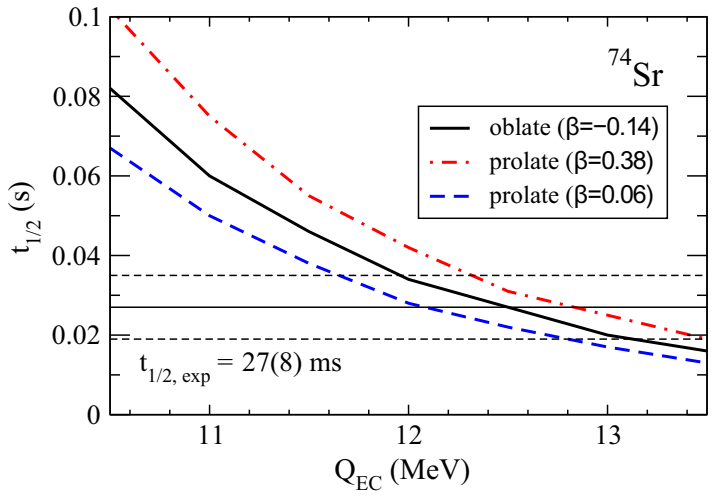

FIG. 4. (Color online) Half-life for ${ }^{74} \mathrm{Sr}$ as a function of $Q_{\mathrm{EC}}$ calculated using a Skyrme (SLy4) Hartree-Fock + BCS + QRPA model [34]. The three curves correspond to the different shape minima predicted for ${ }^{74} \mathrm{Sr}$; the oblate solution is expected to be the lowest in energy and to correspond to the ground state.

interaction, which would mean that the $4^{+}$state in ${ }^{74} \mathrm{Sr}$ was two-proton unbound.

The possible role of Thomas-Ehrman shifts for two-proton bound states has been discussed for light nuclei by Grigorenko et al. [35], while Michel et al. present a detailed discussion of the effects of weak binding in the $A=6$ isobaric triplet [31]. In a sense, these two phenomena are intrinsically linked. Detailed calculations, however, lie outside the scope of the present work, but knowing that these effects are likely to be manifested in the present case does suggest that our present shell-model approach to the evaluation of TEDs may be incomplete when considering the $4^{+}$state.

In conclusion, the recoil- $\beta$ tagging technique was used to identify candidate $4^{+} \rightarrow 2^{+}$and $2^{+} \rightarrow 0^{+}$transitions in ${ }^{74} \mathrm{Sr}$, comprising the first spectroscopic information on excited states in this nucleus. These data have been combined with published information on the $A=74$ isobaric triplet to evaluate TEDs in the heaviest system so far studied. Shell-model calculations involving an isospin nonconserving interaction reproduce TED very well with the exception of $J=4$. The fact that the TEDs for all extant cases may be reproduced with a $J=0$ isospin nonconserving interaction with an isotensor strength of $100 \mathrm{keV}$ strongly suggests that it is a ubiquitous phenomenon mandated across the nuclear chart and has little to no nuclear structure dependence such as the details of orbitals involved in different mass regions. A further observation in the present work is that the half-life of ${ }^{74} \mathrm{Sr}$ is almost twice as short as expected from mass systematics, which suggests that the $4^{+}$state in ${ }^{74} \mathrm{Sr}$ may already be two-proton unbound. This leaves open the intriguing possibility that the position of the $4^{+}$state in ${ }^{74} \mathrm{Sr}$ is perturbed due to effects beyond the present shell-model analysis, for example, Thomas-Ehrman shifts or asymmetry in the wave functions due to weak binding.

This work was supported by the UK Science and Technology Facilities Council, by the Academy of Finland under the Finnish CoE Programme, and by EU-FP7-IA Project ENSAR. The authors acknowledge the GAMMAPOOL European Spectroscopy Resource for the loan of germanium detectors.
[1] P. J. Davies et al., Phys. Rev. Lett. 111, 072501 (2013).

[2] P. Ruotsalainen et al., Phys. Rev. C 88, 041308(R) (2013).

[3] W. E. Ormand and B. A. Brown, Nucl. Phys. A 491, 1 (1989).

[4] A. J. Nichols et al., Phys. Lett. B 733, 52 (2014).

[5] K. Kaneko, Y. Sun, T. Mizusaki, and S. Tazaki, Phys. Rev. C 89, 031302(R) (2014).

[6] A. Steer et al., Nucl. Instrum. Methods A 565, 630 (2006).

[7] J. Henderson et al., J. Instrum. 8, P04025 (2013).

[8] B. S. Nara Singh et al., Phys. Rev. C 75, 061301 (2007).

[9] P. Ruotsalainen et al., Phys. Rev. C 88, 024320 (2013).

[10] H. M. David et al., Phys. Lett. B 726, 665 (2013).

[11] M. Leino et al., Nucl. Instrum. Methods B 99, 653 (1995).

[12] R. D. Page et al., Nucl. Instrum. Methods B 204, 634 (2003).

[13] G. Duchêne et al., Nucl. Instrum. Methods A 432, 90 (1999).

[14] C. W. Beausang, Nucl. Instrum. Methods A 313, 37 (1992).

[15] C. Rossi Alvarez, Nucl. Phys. News 3, 10 (1993).

[16] I. Lazarus et al., IEEE Trans. Nucl. Sci. 49, 567 (2001).

[17] P. Rahkila, Nucl. Instrum. Methods A 595, 637 (2008).

[18] L. F. Sinclair et al. (unpublished).

[19] G. J. Feldman and R. D. Cousins, Phys. Rev. D 57, 3873 (1998).

[20] K-H. Schmidt et al., Z. Phys. A 316, 19 (1984).

[21] G. C. Ball et al., Phys. Rev. Lett. 86, 1454 (2001).
[22] T. Mizusaki, K. Kaneko, M. Honma, and T. Sakurai, Phys. Rev. C 82, 024310 (2010).

[23] T. Mizusaki, K. Kaneko, M. Honma, and T. Sakurai, Acta Phys. Pol. B 42, 447 (2011).

[24] M. Honma, T. Otsuka, T. Mizusaki, and M. Hjorth-Jensen, Phys. Rev. C 80, 064323 (2009).

[25] K. Kaneko, S. Tazaki, T. Mizusaki, Y. Sun, M. Hasegawa, and G. de Angelis, Phys. Rev. C 82, 061301(R) (2010).

[26] K. Kaneko, T. Mizusaki, Y. Sun, S. Tazaki, and G. de Angelis, Phys. Rev. Lett. 109, 092504 (2012).

[27] A. P. Zuker, S. M. Lenzi, G. Martinez-Pinedo, and A. Poves, Phys. Rev. Lett. 89, 142502 (2002).

[28] E. Clement et al., Phys. Rev. C 75, 054313 (2007).

[29] R. G. Thomas, Phys. Rev. 88, 1109 (1952).

[30] J. B. Ehrman, Phys. Rev. 81, 412 (1951).

[31] N. Michel, W. Nazarewicz, and M. Ploszajczak, Phys. Rev. C 82, 044315 (2010).

[32] M. Wang et al., Chin. Phys. C 36, 1603 (2012).

[33] K. Kaneko, Y. Sun, T. Mizusaki, and S. Tazaki, Phys. Rev. Lett. 110, 172505 (2013).

[34] P. Sarriguren, Phys. Rev. C 83, 025801 (2011).

[35] L. V. Grigorenko, I. G. Mukha, I. J. Thompson, and M. V. Zhukov, Phys. Rev. Lett. 88, 042502 (2002). 